\title{
Modeling and synthesis of systems of intensive mass exchange
}

\section{Anatolii Sokolenko, Oleksandr Shevchenko, Kostyantyn Vasylkivskyi, Oleksii Boiko, Anastasiia Shevchenko}

\author{
National University of Food Technologies, Kyiv, Ukraine
}

\section{Keywords:}

Exchange

Intensification

Synthesis

Medium

Inertion

\section{Article history:}

Received 12.03.2019

Received in revised

form 16.07.2019

Accepted 28.11.2019

\section{Corresponding}

author:

Oleksandr

Shevchenko

E-mail:

tmipt@ukr.net

DOI: $10.24263 / 2304-$

974X-2019-8-4-16

\section{Abstract}

Introduction. The development of the theory of synthesis of systems of intensive mass exchange processes at the expense of energy potentials of liquid or gas-liquid flows provides the realization of actual needs.

Materials and methods. The paper analyzes the physical basis of methods and ways of intensification of processes in liquid and gas-liquid systems and phenomenological generalizations based on known laws of nature with mathematical formalization of relationships between geometric, kinematic and hydrodynamic parameters.

Results and discussion. The study shows the possibility of synthesis of inertial fields due to the variable geometric parameters of pipelines, namely, the cross-sectional area, and due to the alternation of curvilinear and straight sections and trajectories of variable curvature.

The generalized representation of the factor of intensification of mass exchange processes relates to force actions in the form of the consequences of pressure redistribution as a result of the redistribution of kinetic and potential energy potential.

Possibilities of variations by values of force influences due to combinations of gravitational field and inertial fields of different orientation and frequency of effects have been shown, including with the possibility of reproduction of dynamic shocks in zones of change of signs of radii of curvature. Synthesis of inertial fields in such systems is achieved by such factors as variable flow velocity or change in flow direction. The combination of these two factors is more effective.

Energy support of the intensification of mass exchange refers to the initial conditions of flow creation, and the overall positive result is ensured by accurate localization of processes.

Conclusions. The factors of intensification of mass exchange processes are represented by the synthesis of inertial fields due to changes in the velocity and direction of the flow of the medium. 


\section{Introduction}

Intensive mass exchange of liquid, gas-liquid or solid-medium media is an important component in a large number of chemical, food and microbiological technologies. Supplementation of mass exchange processes by chemical reactions, destruction or synthesis of substances, changes in chemical, thermodynamic and hydrodynamic parameters in the final result determine the speed of the target processes and the productivity of technological devices. These relationships are reflected in their geometry, total volume, process length etc.

So the cultivation of baker's yeast is carried out on sugar-containing media in machines with a capacity of 70-100 $\mathrm{m}^{3}$. With the same or much larger volumes, the apparatus is used in anaerobic fermentation technologies in the brewery, winemaking, and alcohol industries. In each case, however, there are known limitation factors that make it necessary to use highvolume devices. For example, in aerobic fermentation technologies the processes are inhibited by the activity of yeast, including because of the limited concentration of dissolved oxygen. Anaerobic processes are limited by the osmotic pressure of solutes, etc., but in such cases, the output is sought due to the intensification factors that are realized in the local zones. In this regard, modeling of turbulent flows [1], flows in pipelines with special diaphragms [2], the study of features of transients [3-5], the potentials of mass forces [6, 7] are developed. Theory and numerical analysis allow to use the scientific potential of the Navier-Stokes equations, computational methods in fluid dynamics [8, 9], modern models of hydrodynamics of liquid and gas-liquid media [10-14]. Thus, the applied character of the search for technical solutions determines the relevance of the research and the creation of systems of intensive mass exchange at the expense of internal energy potentials.

\section{Materials and methods}

The paper analyzes the physical basis of methods and ways of intensification of processes in liquid and gas-liquid systems and phenomenological generalizations based on known laws of nature with mathematical formalization of relationships between geometric, kinematic and hydrodynamic parameters.

The object of the study is the technology of intensification of mass transfer processes in systems with limited local zones of interaction [6].

The subject of the research is the factors of intensification as a consequence of the redistribution between potential and kinetic energies in the form of variable kinematic parameters, orientation of flows in the gravitational field, variable pressures, variable geometry of cross sections of flows and curvature of pipeline routes [7].

Methods for investigating the intensification of liquid and gas-liquid systems are related to the use of relationships between kinematic and dynamic flow parameters based on generally accepted mathematical formalizations for straight and curved sections with the determination of dynamic shocks in the zones of change of signs of radii of curvature.

The procedure of the study was determined on the basis of the phenomenological generalization using the laws of nature in the courses of processes with force inertial effects in combination with the effects of the gravitational field.

The final part of the research was related to the analysis of the obtained mathematical models and the formulation of conclusions. 


\section{Results and discussion}

The potential cause of force interaction between solid, gas or liquid fractions and their combinations is the presence of a potential field. So the gravitational field is almost without exception inherent in all technologies of mankind, creating a force of gravity proportional to the mass of the body or medium. The vector characteristic of this field is its overtension $\bar{g}$ in the form of the relation of force $\bar{F}$ to the mass of the object [1,2].

A considerable number of technological processes on the basis of mass and energy exchange in food technologies are carried out in potential fields, due to which the pressure is varied. As for solids, such variations are possible due to the action of external forces and the redistribution of pressures between other bodies at constant indexes of the force potential field. In case of liquid, gas medium (flows) or their combinations, one of two conditions is required [3].

Condition one: Local fluxes are created in liquid, gas or gas-liquid medium in the potential field, in which, according to the Bernoulli and Navier-Stokes equations, the redistribution between potential and kinetic energies and pressures, respectively is carried out. The same redistribution of pressures accompanies the interaction of liquids and gases in the flow of solids, which is accompanied by the occurrence of flows with relative velocities. On this basis, a considerable number of technological methods have been created, which include the use of the aerodynamic profile of the aircraft wing, aircraft screws and helicopters, structures, blades of mixers, ejection devices, etc.

Condition two: the sealing of the local volume of liquid or gas is created and has the manifestation of an increase in the mass quantity of the medium due to external force factors through solids with appropriate energy costs, or the change of the localized sealed volume, or transmission (withdrawal) of heat to the static volume is carried out.

Regarding the first condition, it is noteworthy that the characteristics of the potential field is crucial in the pressure changes. Devices that create artificial force fields based on centrifugal forces are widespread. The principle of their action is based on the movement of curvilinear trajectories with the occurrence of normal accelerations.

The movement of material bodies or flows along curved trajectories means that they have material connection with the centers of curvature in the form of lacs or contact surfaces of curvilinear form.

The force interaction between the streams and the pipes in which they are formed has differences with respect to straight and curved sections. In accordance with the condition of continuity of the flow of the ideal substance in a cylindrical tube with a constant crosssection, the velocity is assumed constant at all points of the latter, and for the uncompressed fluid, the velocity is the same for all sections.

The movement of fluid flows along curvilinear trajectories is accompanied by the creation of fields of centrifugal forces with the action of the latter on each material particle. The combination of centrifugal force and the shell reaction leads to the emergence of a local pressure zone. Since the action and counteraction are mutually compensated, this means that an increased pressure field arises only in the area of the centrifugal force field. The external consequence of the existence of an increased pressure field is an increase in the pressure drop $\Delta \mathrm{P}$ along the length of the curved pipe as the friction forces between the flow and the wall increase. So according to the recommendations [3] pressure loss in the coil pipe is:

$$
\Delta P_{c p}=\Delta P_{s t} \psi,
$$

where $\Delta P_{s t}$ is the loss of pressure on the straight pipe section, and a dimensionless correction factor $\psi>1$ is determined by the formula: 


$$
\psi=1+3.54 \frac{d}{D},
$$

where $\mathrm{d}$ is the internal diameter of the pipe; $\mathrm{D}$ - diameter of the coil pipe.

Due to the given values of kinematic parameters, the tension of the field of inertia forces can significantly exceed the similar potential of the gravitational field. For example, for values of parameters $V=5 \mathrm{~m} / \mathrm{s}, \mathrm{d}=0.1 \mathrm{~m}$ and $\mathrm{D}=0.2 \mathrm{~m}$ we obtain:

$$
a^{n}=\frac{2 V^{2}}{D}=\frac{2 \cdot 25}{0.2}=250 \mathrm{~m} / \mathrm{s}^{2}
$$

and the inertia force from the mass $\mathrm{m}$ in this curved section will be corresponding:

$$
F_{i}=m \frac{2 V^{2}}{D}=250 m, N
$$

In the last entry, the parameter $F_{i}$ is defined as the resultant inertia force corresponding to the distributed pressure. It is important that the transition from the straight-line section to the curvilinear and vice versa is carried out under the condition of instantaneous change of acceleration $a^{n}$, which corresponds to the known phenomenon of soft impact in the dynamics and is estimated as a powerful factor of influence on the system in terms of interests of intensification of energy and mass exchange processes.

These changes in the physical parameters of the impact can have manifestations in limited time or in impulse modes and operate in the extended period of time $[4,5]$.

Given the proportional values of the tensions of the gravitational field and the field of forces of inertia, the orientation of the plane of curvature of the curvilinear trajectory of the flow can have value. Thus, in the horizontal orientation of the system in accordance with the principle of superposition, the total tension of potential fields is determined by their geometric sum:

$$
\bar{a}_{\text {sum }}=\bar{g}+\bar{a}^{n},
$$

which remains constant throughout the site, if $\bar{a}^{n}$ is constant. In the vertical orientation of the specified plane, the values $\bar{a}_{\text {sum }}$ will be variable (Figure 1).

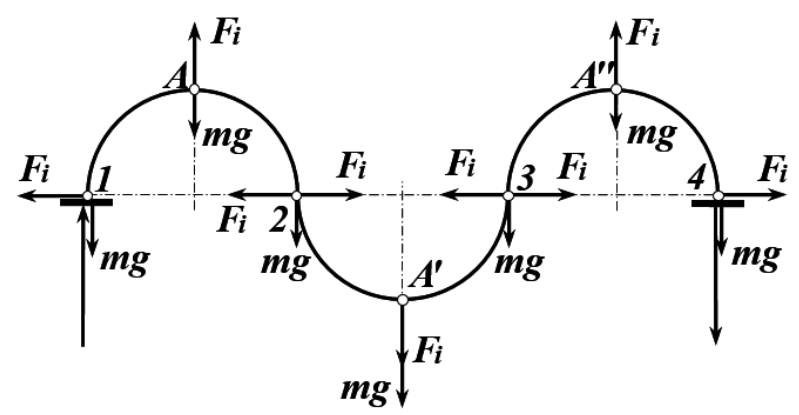

Figure 1. Scheme for determination of inertial force effects on a curvilinear section of a transport system with points of inflection of the trajectory 
Along the whole trajectory, we obtain variable values of mass forces due to different combinations of directions $\bar{F}_{i}$ and $\overline{m g}$. In the case of a route with points of inflection of curvilinear sections with constant values of the radii of curvature, the forces of inertia remain constant, but their directions change by $180^{\circ}$, which leads to the redistribution and action of friction forces between the flow and the pipeline and to changes in the hydrodynamic regime. Changing the direction of inertia forces relative to the flow plays the role of an intensification factor, which is especially important for combined media with the presence of liquid, gas and solid fractions.

Figure 2 shows the marking points of flow $A$ and $B$ and the directions of the resultant gravitational and inertial forces. As we can see, in the closed loop, the result of the gravitational force of unit mass $m g$ on the sections of rectilinear displacement 2-3 and 4-1 relative to the flow has a multi-directional orientation, remaining vertical, and on the sections of curvilinear displacement, the unit mass rotates about $180^{\circ}$ by vector $m g$. Such change in the relative position corresponds to a transient process which additional perturbations occur due to the occurrence of $F_{i}$ inertia forces at points 3 and 1 (Figure 2).

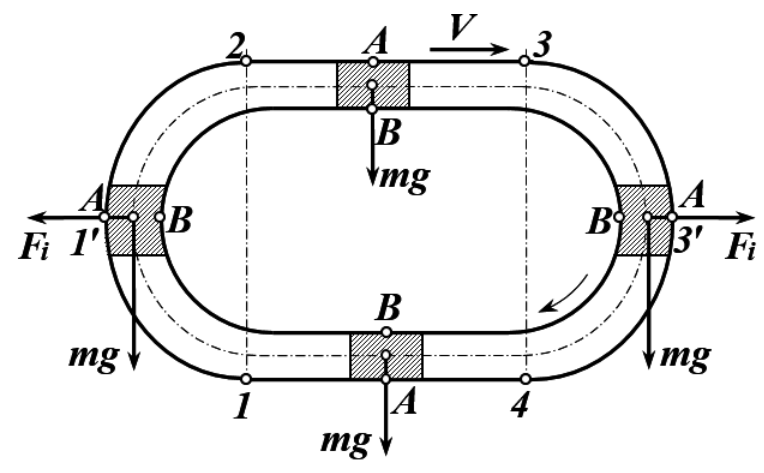

Figure 2. Scheme to determine the directions of force actions relative to the flow

The resultant $R$ is determined by the expression:

$$
\bar{R}=\bar{F}_{i}+\bar{m} \bar{g}
$$

Since the orientation of the resultant of inertia forces $F_{i}$ is related to the position of the radius vector $\bar{r}$, we determine its projections on the coordinate axis of the $O X Y$ system (Figure 3):

$$
\begin{gathered}
F_{i X}=F_{i} \sin \phi=F_{i} \sin \omega t ; \\
F_{i Y}=F_{i} \cos \omega t
\end{gathered}
$$

where $\omega$ is angular velocity of single mass on a curved section; $t$ - the time of movement of single mass in a curved section.

From the plan of forces (Figure 3 ) it can be seen that the resultant projection on the axis $O Y$ for the module is $\left(m g-F_{i} \cos \omega t\right)$. 


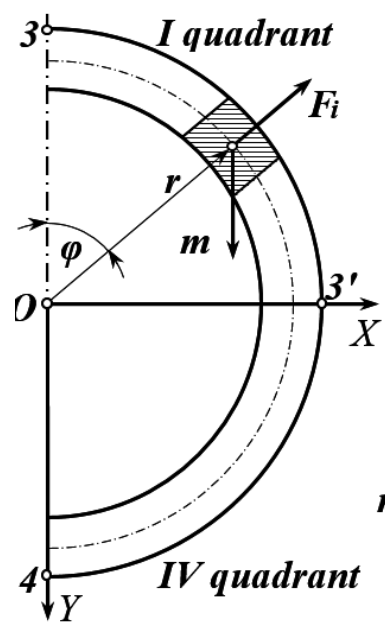

\section{Plan offorces}

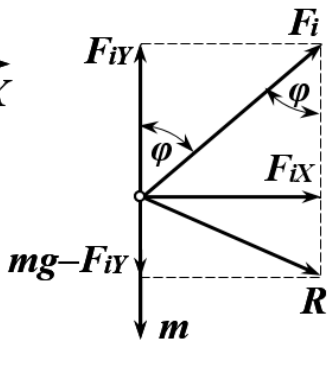

Figure 3. Scheme to determine the force

Then the value of the total resultant for the first quadrant will be

$$
R^{I}=\sqrt{F_{i}^{2} \sin ^{2} \omega t+\left(m g-F_{i} \cos \omega t\right)^{2}},
$$

and for the fourth quadrant -

$$
R^{I V}=\sqrt{F_{i}^{2} \sin ^{2} \omega t+\left(m g+F_{i} \cos \omega t\right)^{2}} .
$$

The maximum value of the resultant in the first quadrant:

$$
R_{\max }^{I}=\sqrt{F_{i}^{2}+(m g)^{2}}
$$

and in the fourth quadrant $-R_{\max }^{I V}=m g+F_{i}$. (10)

At position 4 there is a $R^{I V}$ jump to reduce the force to the value of $m g$, which corresponds to the mode of soft impact. Only $m g$ is valid in section 4- 1 of the mass force, and co-directed with the inertia force $F_{i}$ in point 1 in shock mode. Calculating the angle $\varphi=$ $\omega t$ from the vertical axis in the third quadrant, we determine:

$$
R^{\amalg}=\sqrt{F_{i}^{2} \sin ^{2} \omega t+\left(m g+F_{i} \cos \omega t\right)^{2}},
$$

and for the second quadrant we obtain

$$
R^{I I}=\sqrt{F_{i}^{2} \sin ^{2} \omega t+\left(m g-F_{i} \cos \omega t\right)^{2}}
$$

Choosing a pipeline route in the shape of, for example, a sinusoid wave leads to variable radii of curvature [7]. 
It is known that the general sinusoidal dependence corresponds to the equation

$$
y=A \sin \left(\omega_{0} x+\varphi_{0}\right)
$$

where amplitude $A>0$ and circular frequency $\omega_{0}>0$.

This is a periodic function with period $T=2 \pi$ (Figure 4), the graph of which intersects the $O X$ axis at points with coordinates $(n \pi ; 0)$ ( $n$ is any integer). The points of inflection of the curve correspond to these coordinates. The tangents at these points form, with the positive direction of the axis $O X$, the angles $\pi / 4$ or $-\pi / 4$.

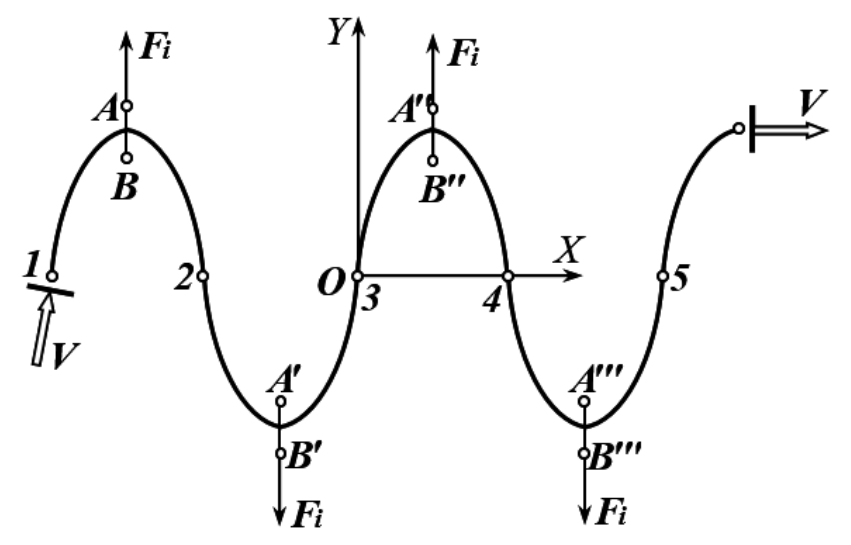

Figure 4. Scheme to determine the power parameters for the sinusoidal line

It is known that the curve $\mathrm{y}=\mathrm{y}(\mathrm{x})$ is determined by the dependence:

$$
K=\frac{y^{\prime \prime}(x)}{\left(1+\left(y^{\prime}\right)^{2}\right)^{3 / 2}},
$$

and the radius of curvature is

$$
r=\frac{1}{K}=\frac{\left(1+\left(y^{\prime}\right)^{2}\right)^{3 / 2}}{y^{\prime \prime}(x)}
$$

With the possibility of free transfer of the origin of the coordinate system OXY, we assume $\varphi 0=0$. Then

$$
\begin{gathered}
y=A \sin \omega_{0} x \\
y^{\prime}=A \omega \cos \omega_{0} x \\
y^{\prime \prime}=-A \omega^{2} \sin \omega_{0} x .
\end{gathered}
$$


Substituting values $y^{\prime}$ and $y^{\prime}$ we obtain

$$
r=\frac{\left(1+A^{2} \omega_{0}^{2} \cos ^{2} \omega_{0} x\right)^{3 / 2}}{-A \omega_{0}^{2} \sin \omega_{0} x}
$$

Let's determine the value of the radii of curvature at the inflection points and extrema. It should be noted that the complex in the numerator of the last dependence will be positive for all values of $x$, since the cosinus function is squared. This means that the sign of the radius of curvature depends only on the sign of sine, namely: in the first and second quadrants the radius is negative, and in the third and fourth - positive.

It should be noted that the notion of the positive and the negative value of the radius of curvature refers to convex and concave parts of a sine wave with respect to the chosen coordinate system, although from a physical point of view the radius of curvature cannot be negative. Figure 5 shows a general view of sinusoidal function and radii of curvature.

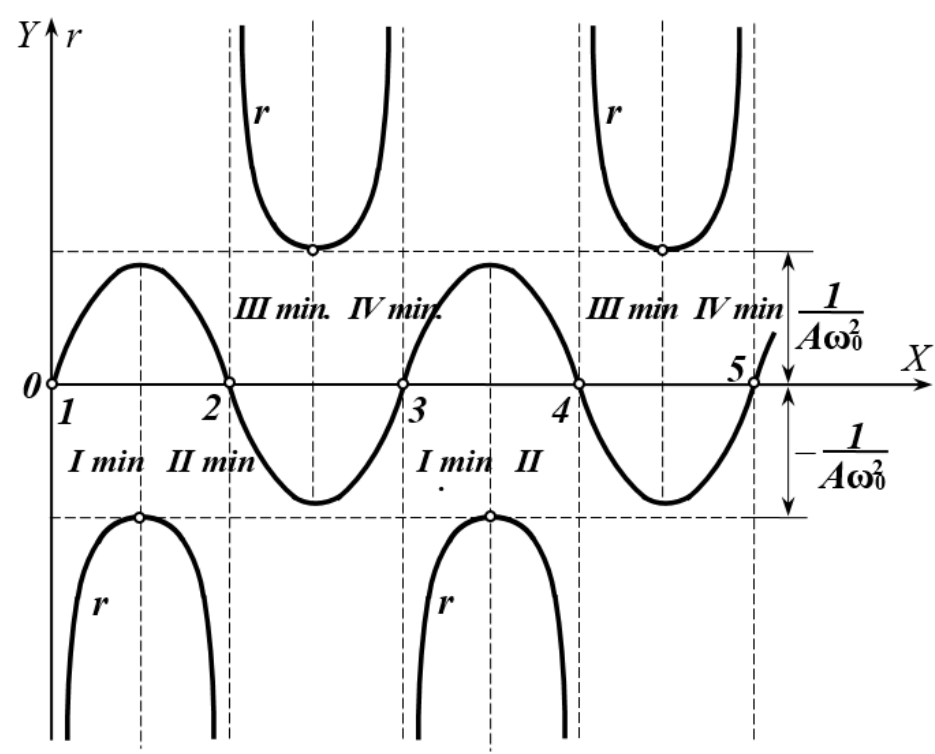

Figure 5. Scheme for determining the radii of curvature of a sinusoid

A change in the direction of the centrifugal force of inertia $F_{i}$ corresponds to the discontinuity at the inflection points of the function $r=r(x)$.

From the point of view of increasing the power flow

$F_{i}$, it is advisable to have as little value of $r$ as possible, since

$$
F_{i}=m \frac{V^{2}}{r}
$$


The value $\omega_{0} x=\pi / 2$ and $\omega_{0} x=3 \pi / 2$ correspond to the extremes of dependence $r=$ $r(x)$ and there is $r=-\frac{1}{A \omega_{0}^{2}}$ and $r=\frac{1}{A \omega_{0}^{2}}$ respectively. At inflection points, we obtain $|r|=\infty$ and $F_{i}=0$.

Thus, in the case of a sinusoidal pipeline route, the inertia force acting on a mass unit $m$

$$
F_{i}=m \frac{-V^{2} A \omega_{0}^{2} \sin \omega_{0} x}{\left(1+A^{2} \omega_{0}^{2} \cos ^{2} \omega_{0} x\right)^{3 / 2}}
$$

is variable by magnitude and direction.

The methods of dynamic influences on liquid or gas-liquid are considered to be worthy of attention and are proposed for implementation, but in the extreme case the situation corresponding to a hydraulic shock should be considered.

Recognizing the changes in pressure as an important factor of intensification of massexchange processes in gas-liquid systems or systems with the addition of solid fraction, we conclude that the deformation is due to the presence of a dispersed gas fraction. This means activation in the interphase surface renewal, coalescence, re-dispersion, vapor phase formation and its collapse at the level of cavitation phenomena. It is important that all these phenomena and transformations occur in conjunction with the operations for transporting the medium. Achieving this result is possible through the use of mass exchange pipelines with variable dimensions and cross-sectional areas. One such option corresponds to the case of successive alternation of transitions from circular to elliptical sections. However, it is obvious that there is an option in which there are simultaneously variable cross-sectional area and trajectory of variable curvature [10].

Let's turn to the estimates of dynamic force manifestations in the latter case. The fundamental value in estimating the parameters of hydrodynamics is the interaction between the streams and the shells that form them. Such determination can be made on the basis of Euler's theorem about the change of the principal vector of quantities of motion of a system of material points. According to it, the sum of the major vectors of the bulk and surface forces, as well as the vectors of the second quantities of fluid flow through two sections of the pipe is zero if the vectors of the second quantities of motion are directed inside the selected cross-sections by volume [12,13].

Let's turn to the example of the flow of fluid through a curved tube, the straight sections of which form an angle of $120^{\circ}$ with velocity w, aiming to determine the additional dynamic pressure of the flow on the walls of the tube. The calculation scheme of this case is shown in Figure 6 in plan. The bulk force is the gravitational force of the fluid, which is perpendicular to the plane of the drawing. The reactions of the wall of the tube, applied to the particles of water in accordance with the law of equality of action and counteracting by the largest vector of the reaction shell are superior.

In the selected coordinate system, the $X$-axis is directed horizontally to the right and the $Y$-axis is vertically up. The vectors of the instantaneous quantities of the flow flowing through the sections 1 and 2 are depicted pointing them inside the volume (Figure 6b). Secondary mass of water is:

$$
m_{c}=\rho f w,
$$

where $f$ is the cross-sectional area of the tube. 


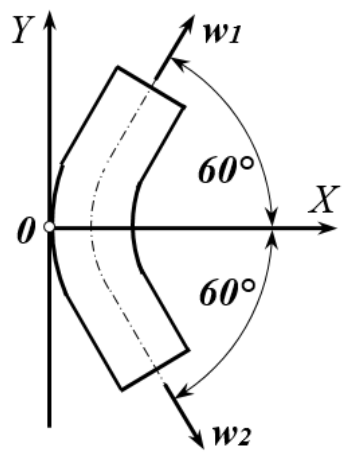

$a$

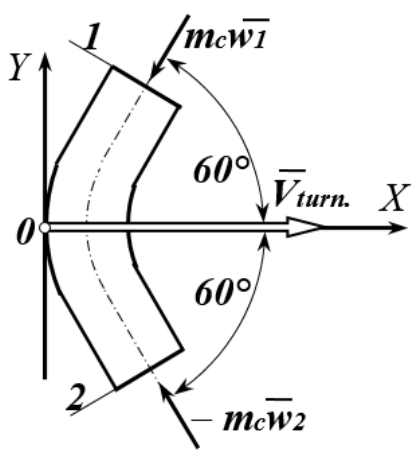

b

Figure 6. Calculation scheme for the case of determination of dynamic flow pressures

Using the Euler theorem, we write down the parameters of the system in the projection on the axis of the selected coordinate system:

$$
\begin{aligned}
& V_{\text {shell.x }}+V_{\text {turn.x }}-m_{c} w_{1 x}-m_{c} w_{2 x}=0 \\
& V_{\text {shell.y }}+V_{\text {turn. } y}+m_{c} w_{1 y}-m_{c} w_{2 y}=0 .
\end{aligned}
$$

In our case vector $\bar{V}_{\text {shell }}$ is perpendicular to the axes $X$ and $Y$, and therefore the system of equations (21)-(22) will be written in the form:

$$
\begin{aligned}
& V_{\text {turn. } x}-m_{c} w \cos 60^{\circ}-m_{c} w \cos 60^{\circ}=0 \\
& V_{\text {turn.y }}-m_{c} w \cos 30^{\circ}+m_{c} w \cos 30^{\circ}=0,
\end{aligned}
$$

where indexes at speeds are not written because $\left|w_{1}\right|=\left|w_{2}\right|=w$.

From this we get:

$$
V_{\text {turn.x }}=2 m_{c} w \cos 60^{\circ} ; \quad V_{\text {turn.y }}=0 .
$$

Thus, the main vector of additional dynamic reactions of the walls of the tube is directed parallel to the axis $O X$. As $m_{c}=\rho f w$, then

$$
V_{\text {turn.x }}=2 \rho f w^{2} \cos 60^{\circ}, \mathrm{N} \text {. }
$$

The main vector $\mathrm{N}$ of forces of additional dynamic pressures on the walls of the tube is equal by modulus and in opposite by direction $V_{\text {turn. }}$, that is, horizontally to the left. This additional component acts only on the curved section and its largest value corresponds to the $180^{\circ}$ rotation angle:

$$
V_{\text {turn.max }}=2 \rho f w^{2}, \mathrm{~N} \text {. }
$$

Such additional force interaction between flow and shell is perceived by the medium as 
an inertial component, the change of which is reflected in the Froude, Reynolds and Euler criteria. Thus, the flow of curvature-stretching sections should be attributed to the number of factors of intensification of mass exchange processes, and acceleration jump in magnitude $w^{2} / r$ and additional dynamic loading in the form of a soft impact correspond to the transition zone from the curved section to the straight line. It is important that such impacts can be fully dosed and programmed.

\section{Conclusion}

The analysis of the peculiarities of mass exchange processes in liquid and gas-fluid systems and flows allows to note the following.

1. The generalized representation of the factor of intensification of mass exchange processes relates to force actions as a consequence of the redistribution between potential and kinetic energies in the form of alternating pressure.

2. Characteristics of a set of potential gravitational fields and artificially generated inertial fields are important in creating alternating pressure. The latter with different characteristics are synthesized at the expense of curvilinear trajectories of flows, which means the presence of their material connection with the centers of curvature in the form of surfaces of contact of curvilinear form.

3. Transitions from curvilinear to rectilinear sections of flow are accompanied by the phenomena of soft dynamic shocks.

4. The intensification factor of mass exchange processes is the geometry of pipeline with variable cross-sectional area. One of the possibilities of realizing such geometry concerns the alternation of circular and elliptical sections due to the corresponding residual deformations.

5. The displacement of flows by curvilinear sections is estimated as a factor of intensification of mass exchange processes, and variable radii of curvature of the route mean the realization of continuously acting influences of inertia forces with manifestations of dynamic shocks in the zones of change of signs of radii of curvature.

\section{References}

1. Belov I., Isaev S. (2001), Modelirovanie turbulentnykh techenii: uchebnoe posobie, Sankt-Peterburg.

2. Ganiev R. (2009), Analiz techeniia v truboprovode so standartnoi diafragmoi sredstvami vychislitelnoi gidrodinamiki, Kazan.

3. Piddubnyi V. (2008), Naukovi osnovy i aparaturne oformlennia perekhidnykh protsesiv kharcho-vykh i mikrobiolohichnykh vyrobnytstv, NUFT, Kyiv.

4. Roache P (1976), Computational Fluid Dynamics, Hermosa, Albuquerque

5. Sokolenko A., Piddubnyi V., Ukrainets A. (2007), Vykorystannia potentsialu masovykh syl dlia intensyfikatsii masoobminu v ridynnykh i hazoridynnykh potokakh, Kharchova i pererobna promyslovist, 3, pp. 15-17.

6. Shevchenko O.(2006), Naukovi osnovy $i$ aparaturne oformlennia protsesiv dovhostrokovoho zberihannia kharchovykh produktiv, NUFT, Kyiv.

7. Sokolenko A., Khomenko M., Shevchenko O. (2007), Masoobmin u neliniinykh truboprovodakh, Kharchova i pererobna promyslovist, 5, pp. 20-21. 
8. Temam Roger (1979), Navier-Stokes Equations. Theory and Numerical Analysis. 2nd Edition, North Holland.

9. Fletcher K. (1991), Vychislitelnye metody v dinamike zhidkostei. T. 1, Mir, Moscow.

10. Frik P. (1998), Turbulentnost: modeli i podkhody, Perm.

11. Anderson John D., Jr. (1995), Computational Fluid Dynamics: The Basics with Applications, McGraw-Hill, New York.

12. Bajura R., Pellgrin M. (1977), Studies of pulsating incompressible flow through orifice meter, U. S. Dep. Commer. Nat. Bur. Stand. Spec. Publ, 84/2, pp.523-548.

13. Harvard Lomax, Thomas H. Pulliam, David W. Zingg (1999), Fundamentals of Computional Fluid Dynamics, University of Toronto Institute for Aerospace Studies, Toronto.

14. Hofmann G. (1996), Flotation Die Technik der Zukynf, Der Deutsche Weinbau, 1, pp. 20-21. 